Meta

Journal des tradlucteurs

Translators' Journal

\title{
La valeur relative des définitions comme référence terminologique
}

\section{J. Albert Bachrach}

Volume 18, numéro 1-2, mars 1973

Actes du deuxième colloque international de linguistique et de traduction. Montréal, 4-7 octobre 1972

URI : https://id.erudit.org/iderudit/001941ar

DOI : https://doi.org/10.7202/001941ar

Aller au sommaire du numéro

Éditeur(s)

Les Presses de l'Université de Montréal

ISSN

0026-0452 (imprimé)

1492-1421 (numérique)

Découvrir la revue

Citer cet article

Bachrach, J. A. (1973). La valeur relative des définitions comme référence terminologique. Meta, 18(1-2), 161-170. https://doi.org/10.7202/001941ar d'utilisation que vous pouvez consulter en ligne.

https://apropos.erudit.org/fr/usagers/politique-dutilisation/ 


\section{La valeur relative des définitions comme références terminologiques}

Les problèmes de la traduction et de la terminologie suscitent actuellement beaucoup d'intérêt dans les milieux les plus divers. Sans vouloir m'étendre sur les motifs de cet intérêt généralisé, il faut que je m'en félicite en tant que linguiste car ceux qui autrefois ne reconnaissaient guère l'existence des barrières linguistiques commencent aujourd'hui à se rendre compte des problèmes qu'elles suscitent.

S'il y a lieu de craindre le chômage dans beaucoup de professions, ce danger ne semble pas menacer les linguistes, fussent-ils traducteurs, interprètes, lexicologues ou terminologues. Je suis personnellement bien placé pour constater combien il est difficile de trouver des éléments valables et cela, tant au point de vue des connaissances linguistiques qu'à celui de leurs connaissances en ce qui concerne le volet considérable des domaines d'activité des grandes organisations européennes et mondiales.

Dans le chapitre du tome le Langage de 1'«Encyclopédie de la Pléiade », consacré par Jean-Paul Vinay à la traduction humaine, est cité en conclusion Roger Caillois qui estime que bien traduire signifie écrire le texte aussi bien que l'aurait produit un auteur donné s'il avait disposé des ressources de la langue d'arrivée du traducteur au lieu de celles de sa propre langue. Autrement dit, le traducteur doit véritablement recréer le texte de l'auteur traduit sans pour autant l'interpréter à sa façon.

On pourrait croire que cette thèse n'est valable que dans le cas de traductions de textes littéraires. Mais cela n'est point le cas, car le développement du langage propre à une technique ou discipline données oblige le traducteur à se soucier de la façon dont s'expriment les experts de la matière lorsqu'ils emploient la langue de travail du traducteur. En outre, il est indispensable que la traduction fasse preuve d'une grande précision : des libertés d'expression, ou plutôt « 1'à-peuprès », ne sont en général pas admis.

Pour atteindre cette précision, il convient de rechercher la signification des termes inconnus ou dont on ignore le sens exact. Inutile de souligner que l'on ne peut guère se fier uniquement aux dictionnaires, même lorsqu'ils sont réputés «spécialisés ». Dans de nombreux cas, des recherches plus approfondies devront être entreprises afin de vérifier si la traduction relevée dans le dictionnaire convient au contexte qui doit être traduit. $\bar{A}$ ce stade, beaucoup de linguistes estiment que seule la vérification des définitions peut fournir des résultats concluants. Or, 
avant d'accepter une telle affirmation, il convient d'examiner le caractère de la définition et son objectif.

En consultant les dictionnaires unilingues courants, nous constatons qu'il existe plusieurs genres de définitions. Le Petit Robert fait mention de la définition exacte ou fausse et de la définition caractéristique qui comprend une comparaison avec un autre concept qui, toutefois, est équivalent. Cette définition énumère donc tous les aspects communs et toutes les différences qui existent entre eux. En ce qui concerne le domaine de la linguistique, il s'agit, d'après ce dictionnaire : « d'une phrase qui définit un élément du lexique (mot, expression) ; une formule brève correspondant à un concept raisonnable et capable d'en susciter l'élaboration. Définition d'un mot dans un sens ». Je ne m'étendrai pas davantage sur les autres parties du chapitre du Petit Robert. Toutefois, j'aimerais relever qu'en second lieu il y est fait mention de l'action de caractériser, c'est-à-dire la description. Une liste est consacrée au terme technique « définition» qui, dans le domaine de la télévision, revêt un sens tout à fait particulier.

Le chapitre que le Larousse consacre à « définition» nous apprend qu'il s'agit de «l'énonciation des qualités essentielles d'un objet $»$. Ensuite, nous lisons encore : «explication, signification : la définition d'un mot, d'une expression》. Pour le reste, cet ouvrage ne donne que de très succinctes indications quant aux définitions dans la logique. Mais à la différence du Petit Robert, la signification du terme technique dans le domaine de la télévision y est amplement détaillée.

Les définitions de termes techniques faisant l'objet des travaux des instituts de normalisation, nous nous sommes ensuite penchés sur diverses recommandations de l'I.S.O. (Organisation internationale de normalisation et, plus particulièrement, sur celles qui ont été élaborées par son Comité technique I.S.O./TC/37 Terminologie (principes et coordination), dont le secrétariat est assuré par l'Institut de normalisation autrichien (Österreichisches Normungsinstitut). Dans le vocabulaire de la terminologie, publié dans la recommandation I.S.O./R/2087-1969, figurent quatre genres de définitions, notamment $: a$ ) la définition qui égale la détermination verbale d'une notion; $b$ ) la définition spécifique ou définition au sens classique, c'est-à-dire la détermination de la compréhension d'une notion; c) la définition générique, à savoir la détermination de l'extension d'une notion; et enfin $d$ ) la définition par l'emploi : en d'autres termes, la définition sous forme d'un exemple d'emploi, c'est-à-dire d'une équation implicite. Pour ce genre de définition, le terme est présenté dans une phrase dont la signification entière est connue ou peut être devinée.

L'utilisateur d'une définition quelconque devra donc, en premier lieu, s'assurer du genre de définition dont il aura besoin. Il devra ensuite également être capable de vérifier si la définition qu'il aura trouvée appartient vraiment au type recherché.

Les normalisateurs soulignent généralement l'importance de l'usage d'un terme qu'ils ont défini de préférence à un autre désuet ou non consacré dans une norme. Toutefois, ils conviennent, et la recommandation sur les principes de dénomination ${ }^{1}$ en fournit la preuve, que $\ll$ les définitions génériques, contrairement

1. I.S.O./R/704-1968, 2.2.3.1. 
aux définitions spécifiques, définitions au sens classique, ne peuvent être exhaustives car de nouvelles espèces de la notion à définir peuvent être découvertes ou inventées après l'établissement de la définition générique ». Pour ma part, je suppose que tel a été le cas pour la détermination de la signification du terme «définition» dans le domaine de la télévision, où l'on distingue même une définition verticale et une définition horizontale, car cette extension de la notion ne peut guère être antérieure à l'invasion de nos foyers par la télévision. La recommandation dont il est question le dit d'ailleurs très clairement : «les définitions génériques varient avec le temps ${ }^{2}$.»

Le traducteur technique et scientifique se trouvera souvent confronté à des définitions qui, même si elles ont été établies dans une même langue et à une même date, ne concordent apparemment pas entre elles, bien qu'elles contiennent une description de la même notion et du même terme.

$\mathrm{Si}$ cette constatation peut être faite pour une seule langue, combien plus fréquents seront les cas où les définitions varient considérablement d'une langue à une autre. L'I.S.O. a d'ailleurs bien reconnu ce fait. Aussi a-t-elle préconisé l'unification de l'énoncé des définitions. Dans sa recommandation au sujet de l'unification internationale des notions et des termes ${ }^{3}$, le vocabulaire de la Commission électrotechnique internationale (C.E.I.) est donné comme exemple. L'énoncé officiel des définitions (en anglais et en français) est établi, tandis que «des traductions de cet énoncé-modèle doivent ensuite être introduites au fur et à mesure dans les normes nationales ».

Cette même recommandation met également en garde contre "l'introduction dans un pays particulier, de définitions fixées par une convention internationale sans examen critique préalable», car ces définitions sont souvent établies sur des bases répondant plus spécialement aux usages de certains autres pays.

Puisque l'objectif essentiel des traductions est de faciliter l'information et la communication, il me semble intéressant de vous exposer ce que j'ai relevé en matière de définition de la notion «information » en vous avertissant cependant que mes recherches sont forcément loin d'être exhaustives. Voici donc ce que nous propose le Petit Robert pour le mot « information»:

$1^{\circ}$ « Ensemble des actes qui tendent à établir la preuve d'une infraction et à en découvrir les auteurs »; comme synonyme il y a d'abord un renvoi à «instruction » : * ouvrir une information, information officielle, officieuse *; puis à «enquête ».

$2^{\circ}$ "Renseignements sur qqn, sur qqch», et «d'utiles informations», avec comme renvoi * tuyau ", assorti de la locution " je suis venu aux informations " (citation extraite des œuvres littéraires de Marcel Aymé) et de l'indication d'un sens nouveau e ensemble des renseignements obtenus par qqn., une information prodigieuse ».

$3^{\circ}$ "Action de s'informer, de prendre des renseignements" et un nouveau renvoi à "enquête, examen, investigation ", ainsi que la locution " homme politique en voyage d'information " et comme dernier renvoi "étude».

$4^{\circ}$ « Renseignement ou événement qu'on porte à la connaissance d'une personne, d'un public», avec le renvoi à "nouvelle» notamment «informations politiques, sportives, bulletin d'informations"; et ensuite le renvoi à "communiqué, journal (parlé, télévisé), une information sensationnelle $x$.

2. I.S.O. $/ \mathrm{R} / 704-1968,2.2 .3 .1$.

3. I.S.O./R/860-1968, 2.1 . 
$5^{\circ}$ ( $\mathrm{xx}$ siècle) : * Ensemble des informations * et, par extension, "action d'informer le public, l'opinion; agence d'information, information et propagande; journal d'information - techniques d'information : presse, radio, cinéma, télévision ».

$6^{\circ}$ Nous trouvons ici quelques indications quant à la signification du mot dans le domaine scientifique à partir de 1950 : «Élément ou système pouvant être transmis par un signal ou une combinaison de signaux (renvoi à message), ce qui est transmis (objet de connaissance, mémoire) ». Ensuite, mention est faite de "théorie, traitement de l'information (informatique); information et cybernétique ». Puis une locution empruntée à une publication de l'académicien De Broglie : "une machine à calculer peut communiquer à des utilisateurs les résultats de ses calculs, c'est-à-dire de l'information». Nous apprenons enfin que, dans un autre domaine, on parle "d'information génétique ": "caractères héréditaires transmis par les gènes ».

Dans tout ce chapitre, ce qui me frappe le plus est que l'accent y est mis sur les significations que peut revêtir le terme «information» dans le langage juridique, d'une part, et dans la presse en son sens le plus large, de l'autre.

D'ailleurs, si nous consultons le Larousse, nous y rencontrons ce même phénomène. Jacques Arsac a déjà attiré l'attention sur ce fait dans son ouvrage la Science informatique ${ }^{4}$ lorsqu'il constate qu'à l'origine «information » est un terme de justice, mais qui déjà porte en lui l'action d'obtenir des renseignements, d'acquérir des connaissances. Il propose d'affiner la définition en parlant d'apport éventuel de connaissance. Avec cette précision, il s'approche visiblement de la phrase citée dans le Petit Robert « communiquer... de l'information ».

À l'occasion d'une recherche que j'ai commencée avec l'assistance d'une collaboratrice, nous avons retrouvé cette même définition, à savoir : « connaissance qui peut être communiquée $»$, dans la publication de l'U.N.E.S.C.O. (Draft Terminology of Documentation ${ }^{5}$ ). Encore faut-il préciser que la publication citée indique comme synonymes de ce terme « renseignement » et, par extension, «processus d'information ». Ceci explique l'extension à la définition : «signification que l'homme attribue à des données à l'aide des conventions employées pour les représenter 》 et à «accroissement de la connaissance, c'est-à-dire, réduction de l'incertitude à l'aide d'une communication», pour ensuite arriver au «processus de communication des informations \$.

Mais voyons à présent ce que nous avons trouvé en anglais. Bien sûr, nous rencontrons en tête de liste information suivie de knowledge, data, meaning, information process. Les définitions anglaises correspondent bien à celles que je vous ai citées en français, à savoir :

"Knowledge that can be communicated" (1);

"Communicated knowledge, i.e., signs representing knowledge " (2) ;

" The meaning that a human assigns to data by means of the conventions used in their representation $\star(3)$;

- The process of communicating information * (4).

Ces termes et leurs définitions ont été puisés dans les publications de l'U.N.E.S.C.O., de l'I.S.O. et de l'I.F.I.P. ${ }^{6}$.

Toutefois, toutes ces définitions n'enlèvent rien à l'ambiguité du terme «information», même dans la définition de De Broglie que je reprends ici :

4. Jean Arsac, la Science informatique, Paris, Dunod, 1970, 2.3.

5. Draft Terminology of Documentation, E.F.D., U.N.E.S.C.O., 1971.

6. I.F.I.P. (International Federation for Information Processing). 
《une machine à calculer peut communiquer à des utilisateurs les résultats de ses calculs, c'est-à-dire, de l'information. " Se référant aux définitions citées, le profane peut, en effet, se demander si le terme «information» se rapporte aux 《 résultats de ses calculs », à «communiquer», ou à l'《 action de calculer ». Le transfert de l'information résulte en fait d'une ou plusieurs opérations qui pourrailent être considérées comme synonymes d'une enquête.

Le traducteur averti fera sans doute le lien avec «informatique», terme qui n'a pas été jugé digne de figurer comme mot clé dans le Petit Robert de 1967. Le Larousse, par contre, lui consacre une définition succincte, à savoir : «technique du traitement automatique de l'information.»

Ce terme a d'ailleurs donné lieu à l'élaboration de trois définitions qui diffèrent quelque peu entre elles : $a$ ) celle de l'I.S.O. qui constate que l'informatique englobe toutes les disciplines scientifiques et techniques qui peuvent spécifiquement servir au traitement de l'information par des moyens automatiques; b) celle élaborée par le Comité terminologique de l'Académie française qui diffère du texte de l'I.S.O. pour autant que l'accent y est mis sur le traitement rationnel de l'information, considérée comme le support des connaissances dans divers domaines ; $c$ ) enfin, nous avons trouvé dans l'ouvrage l'Informatique de Delagrave qu'il s'agirait plus particulièrement de la technique (connaissances, principes, méthodes et moyens) du traitement logique et automatique de l'information.

Pour les autres langues (je me bornerai ici à l'anglais), nous avons constaté qu'en ce qui concerne le terme informatics, l'I.F.I.P. ${ }^{7}$ donne une définition plus restrictive : "those aspects of science and technology specially applicable to data processing and to automatic data processing». Dans le sens de la définition française de l'I.S.O., cette même organisation nous propose comme équivalent d'informatique automatic data processing dont la définition anglaise correspond parfaitement à la formulation française de Delagrave. "That branch of science and technology concerned with methods and techniques relating to data processing largely performed by automatic means. »

Le terme informatics semble d'ailleurs avoir été défini pour la première fois en 1966 dans le rapport de trois scientifiques russes : "The new scientific discipline which studies the structure and properties of scientific information as well as the regularities of scientific information activity, its theory, history, methods,

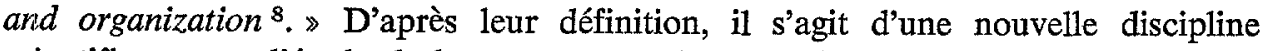
scientifique pour l'étude de la structure et des caractères de l'information scientifique, la régularité des activités dans le domaine de l'information scientifique, la théorie, l'historique, les méthodes et l'organisation.

En France et en République fédérale d'Allemagne, les termes « informatique 》 et informatik ne sont guère employés en ce sens. Le terme anglais informatics aurait

$\overline{7 .}$ I.F.I.P.-I.C.C., Vocabulary of Information Processing, Amsterdam, 1968 (Joint Technical Committee on Terminology : International Federation for Information Processing and International Computation Centre); I.F.I.P., Guide to Concepts and Terms in Data Processing, édit. par I .H. Gould, Amsterdam, 1971.

8. Informatics (New Name for the Theory of Scientific Information), U.R.S.S., Mikhailov, Chernyi et Cilyarevskii, 1966. 
plutôt comme équivalent «science de l'information scientifique » tel qu'il figure dans la terminologie déjà mentionnée de l'I.F.I.P. L'U.N.E.S.C.O. propose comme équivalent anglais : "informatique »= computer science en donnant la définition : «science concerned with the techniques of computer design and application. $\gg$

Toutes ces définitions, si belles et explicites soient-elles, n'enlèvent rien, il me semble, à la difficulté de traduire des phrases telles que : « à condition qu'elle n'aboutisse pas, en définitive, à une perte de contrôle des éléments clés de l'industrie informatique ${ }^{\circ}$ », ou encore : « Depuis février dernier, ces deux sociétés ont en effet décidé d'associer intimement leurs efforts dans le domaine de la recherche, la fabrication et la commercialisation de produits informatiques sur l'ensemble du monde ${ }^{10}$. $»$ Et comment rendre : «dans le secteur de la petite informatique ", locution rencontrée dans le même article dont sont extraites les deux phrases précédentes du quotidien le Monde ${ }^{11}$.

Dans le cas des «éléments clés de l'industrie informatique», on pourra fort bien hésiter entre key data in the data-processing industry et key data in the computer industry, selon qu'on met l'accent sur le terme « éléments clés » ou sur 1 ' « industrie informatique ». La même hésitation pourra se reproduire au sujet des produits informatiques car ne pourrait-on pas considérer les informations obtenues moyennant un traitement en ordinateur comme des «produits informatiques $» ?$ Le terme «fabrication》 ne l'éclairera guère davantage puisqu'il ne semble pas possible de parler de la fabrication de produits informatiques dans le sens d'une masse d'informations ou de données. Mais on ne manquera point d'observer que la phrase comprend également le terme « recherche », ce qui laisse supposer que les "produits informatiques \& comprennent aussi le software.

Nous proposons donc de traduire «produits informatiques» par computer and data-processing products et «industrie informatique 》 par computer and dataprocessing industry. Les deux termes ont été glanés dans l'ouvrage de Michael Rose, Computers, Managers and Society ${ }^{12}$.

La locution précitée «dans le secteur de la petite informatique»pourrait être rendue par in the sector of the small computers. Nous proposons cependant comme équivalent de «petite informatique », baby computers, terme également employé par Michael Rose dans son livre.

Il n'y a pas de doute que celui qui devra effectuer la traduction inverse, c'est-à-dire rendre en français computer industry, aura bien des difficultés à trouver le terme «industrie informatique» que nous avons en vain cherché dans les ouvrages de référence ou dictionnaires spécialisés.

Le titre de mon exposé et les arguments que je viens de vous soumettre pourraient vous mener à croire qu'à mon avis, les définitions - qu'elles soient élaborées par les organisations de normalisation ou non - n'ont aucune valeur pour le traducteur. Or, ceci n'est nullement le cas.

9. Le Monde, supplément le Sicob, 19 septembre 1972, p. 23.

10. Ibid.

11. Ibid.

12. Michael Rose, Computers, Managers and Society, Pelican Books, 1969, p. 234-236. 
$\mathrm{Vu}$ les mises en garde répétées contre l'emploi sans discernement de dictionnaires, il me paraît utile d'ajouter qu'il faut également se méfier des définitions. En effet, ainsi que je l'ai déjà fait remarquer, celles-ci n'ont généralement pas été conçues dans le but d'enlever toute ambiguïté à un terme, mais plutôt dans l'intention de nommer un objet ou de fixer l'appellation exacte d'une notion. Les normes officielles constituent d'excellentes sources pour la vérification de l'exactitude des termes que le traducteur désire employer ou que le terminologue a l'intention d'insérer dans son fichier.

Les dictionnaires qui ont été élaborés sur la base des normes - je pense plus particulièrement au Dictionnaire multilingue de la machine-outil d'Eugen Wuester ${ }^{13}$ — sont d'une très grande utilité, mais leur nombre est hélas encore trop limité.

Le travail du traducteur ne consiste pas à rendre dans une autre langue des mots isolés, mais des blocs homogènes de mots qui expriment une image ou une iclée. Le traducteur ne pourra donc pas se limiter à la consultation de ce genre de dictionnaires, si précieux soient-ils. Certes, il y puisera des termes techniques très utiles ainsi que des indications concernant ceux dont l'usage n'est plus accepté, mais il y cherchera en vain les néologismes, étant donné que l'élaboration d'un tel ouvrage nécessite a peu près dix ans. Même si les recommandations de I'I.S.O. prévoient des définitions par l'emploi ${ }^{14}$, des définitions de ce genre ne sont que très rarement incluses. D'ailleurs, elles aussi peuvent se démoder et l'exemple donné dans le vocabulaire de la terminologie publié par l'I.S.O., en 1969, à savoir : «il allait d'Europe en Amérique en 24 heures en utilisant un... (aéronef) 》, nous fait sourire trois ans après la publication de ce document. En effet, nos connaissances se sont accrues, nous avons donc subi un apport de connaissances et nous savons, soit par expérience personnelle, soit par oui-dire, qu'aujourd'hui la traversée de l'Atlantique par la voie aérienne ne requiert que six heures à peine.

Le traducteur saura qu'en anglais «la voie aérienne » peut être rendue par air-way ou air-route, mais que dans le cas cité, il vaudra mieux employer l'expression by air ou by plane et non by aircraft, l'équivalent d'aéronef...

Des linguistes, tels que Leo Weisgerber ${ }^{15}$, prétendent que le sens d'un mot se trouve uniquement dans sa définition car chaque mot éveille chez l'homme l'association avec un objet, un concept ou une notion. Ils condamnent vivement la fameuse thèse de Ludwig Wittgenstein ${ }^{16}$ et de ses disciples de Cambridge, selon laquelle le mot ne revêt sa véritable signification qu'à l'intérieur d'une phrase. Or, la pratique de la traduction des langues prouve que dans nombre de cas le contexte seul ouvre la voie vers la bonne solution, car c'est le contexte qui permet de vérifier la signification du terme et en même temps son emploi.

Même si le traducteur dispose d'une mémoire prodigieuse, il ne pourra guère rester au courant du développement du langage technique et scientifique dans

13. Dictionnaire multilingue de la machine-outil, notions fondamentales définies et illustrées par Eugen Wuester, E.F.D., Londres, Technical Press, 1968.

14. I.S.O./R/1087-1969, 2.30 .

15. Leo Weisgerber, Die vier Stufen in der Erforschung der Sprachen, Schwann, Düsseldorf, 1963 , p. 68-69.

16. Ludwig Wittgenstein, A Memoir by Norman Malcolm, p. 93. 
tous ses détails, s'il se réfère uniquement aux définitions qui sont contenues dans les encyclopédies et dictionnaires, ou à celles qui ont fait l'objet des publications des organisations de normalisation. Ces recherches seront vaines dans bien des cas. Les définitions ainsi élaborées sont figées dans l'espace et dans le temps; elles ne peuvent guère tenir compte des évolutions futures. La langue, qu'il s'agisse de la langue courante, technique, scientifique, populaire ou savante, est en constant mouvement et ne fonctionne point d'après des règles préconçues. Il n'existe aucun moyen d'imposer l'emploi de certains termes au détriment d'autres, ni d'interdire aux scientifiques l'emploi de tel calque repris d'une langue étrangère. Les néologismes ne seront pas invariablement créés d'après les directives de l'I.S.O. ou de l'Académie française. N'est-il pas plutôt rare de rencontrer des techniciens ou des scientifiques qui sont en même temps linguistes et qui respectent sans dérogations ces règles?

Afin de pouvoir accomplir sa mission qui consiste essentiellement à rendre possible la communication au-delà des barrières linguistiques, le traducteur doit disposer des outils lui permettant de s'informer au sujet de la signification de tous les termes et de leur emploi. C'est le contexte qui peut dans beaucoup de cas l'aider à décider s'il faut traduire «mémoire» par memory, remembrance, recollection, mind ou information store. La définition du terme technique, si elle existe, l'informera ensuite des différentes sortes de mémoires et de leurs fonctions dans l'ensemble d'un centre de calcul.

Les instituts de normalisation et tous ceux qui s'occupent de normaliser la terminologie en s'efforçant d'élaborer des définitions pour chaque terme, accomplissent une tâche à posteriori, les termes ayant, me semble-t-il, déjà été créés avant la définition.

Par contre, la fonction du traducteur se situe immédiatement après la création des termes. Le traducteur doit donc sans tarder trouver des solutions valables et non pas dix ans après. Il lui faut rechercher pourquoi la communicatique ${ }^{17}$ n'équivaut point à l'informatique mais en est plutôt une émanation. C'est pour cette raison que j'estime que le travail du terminologue se situe entre la traduction et la normalisation. Autrement dit, le terminologue doit aussi bien enregistrer la terminologie d'un domaine précis et découvrir des définitions qui ne sont pas encore officiellement admises par les normalisateurs, que glaner dans les textes - de préférence en langue originale - les néologismes et leur emploi.

Il ne faudra toutefois pas en conclure que la terminologie, même contextuelle, est à considérer comme un but en soi alors qu'elle est un instrument dont peut se servir utilement le traducteur. Les définitions font nécessairement partie de son « environnement».

La terminologie ne doit surtout pas constituer un piège ou une toile d'araignée d'où la traduction ne pourra plus jamais s'extraire.

Par contre, en traduction comme partout ailleurs, l'homme seul ne peut plus aboutir. Les définitions et la terminologie doivent constituer toutes deux des 17. Gérard Métayer, les Systèmes électroniques de la communication. La communication,
Paris, Les Editions d'Organisation, 1972. 
sources d'information qui se complètent et qui font partie intégrante de la panoplie du traducteur. Afin que ce dernier puisse en tirer le maximum de profit, il faudra parvenir à une meilleure mise au point de leur accès, de leur interaction et de la manière dont elles peuvent rapidement et efficacement être communiquées.

J. Albert Bachrach

\section{QUESTIONS}

Mine Josette Rey-Debove : Je remercie $\mathrm{M}$. Bachrach de son exposé et je suis d'accord avec lui pour ainsi dire sur tout. Ses récriminations sont entièrement justifiées. Je le trouve cependant à la fois trop indulgent et trop sévère. D'une part, trop sévère quand il juge des dictionnaires de langues en un volume. Évidemment ces dictionnaires ne peuvent pas donner sur les mots techniques et scientifiques des informations qu'on trouve dans les dictionnaires encyclopédiques en dix volumes. C'est dommage, je le reconnais, mais enfin les traducteurs seraient bien avisés effectivement, quand il s'agit d'un mot technique ou scientifique, d'aller chercher ailleurs, c'est tout de même plus prudent. D'autre part, je trouve que vous êtes indulgent en ce qui concerne les ambitions de la définition. Je ne pense pas que la définition veuille situer l'objet qu'elle définit; je crois qu'elle est plus ambitieuse que cela et elle prétend donner les traits pertinents de l'objet défini par opposition à tous les autres objets. Elle y atteint assez rarement, mais je crois quand même que si elle n'y atteint pas ce n'est pas volontaire; on a vu tout à l'heure des exemples de définitions qui, à mon avis, étaient choisis d'une manière un peu partiale. On avait : rossignol, oiseau qui chante bien, dans le Dictionnaire multilingue de la machine-outil. C'est évident que ce n'est pas une définition, c'est une inclusion, le rossignol fait partie de l'ensemble des oiseaux qui chantent bien; on n'a pas prétendu le définir par là. Mais là je crois que c'est tout à fait spécial, il me semble que les auteurs avoueraient que ce n'est pas une définition. Pour ma part, même dans le cas de dictionnaire de langue, je pense que la définition est beaucoup plus ambitieuse. La définition dans le dictionnaire ne donne pas un signifié, mais elle donne toujours ce qui est désigné. C'est sur le plan de la désignation qu'elle opère, car je ne vois pas comment le lexicographe aurait les moyens de donner le contenu d'un mot alors que les sémanticiens ne peuvent pas le faire.
Réponse : Tout d'abord, je ne crois pas être trop sévère pour les dictionnaires. Je les utilise beaucoup en particulier ceux que j'ai cités. J'ai seulement voulu souligner que le traducteur a besoin encore d'autres outils que ses dictionnaires. Si j'ai parlé des dictionnaires unilingues et surtout des deux dictionnaires français que j'ai mentionnés, c'est parce que nous en sommes des utilisateurs assidus et que je conseille à mes jeunes traducteurs de s'y référer régulièrement lorsqu'ils ne trouvent pas ce qu'ils cherchent dans les dictionnaires multilingues. D'autre part, en ce qui concerne la définition, je me suis seulement basé sur les descriptions que j'ai trouvées, et dans le Larousse et dans le Petit Robert, et les différents principes de définition énoncés par l'X.S.O. Nous avons aussi besoin d'autre chose et très souvent un dictionnaire contextuel ou bien des contextes qu'on a puisés ailleurs dans des textes, dans des descriptions, dans des rapports originaux peuvent donner des indications de bon emploi des termes.

M. Gauger : Je vois que cet exposé a très bien mis en relief quelques-uns des problèmes devant lesquels nous nous trouvons. Justement, je crois qu'il faut faire une différence entre les termes qui appartiennent au langage courant et les termes qui ont un caractère plus technique. Le problème réside évidemment dans le fait que nous trouvons dans le langage technique même beaucoup de mots qui appartiennent au langage courant, mais qui ont aussi un caractère technique. Rossignol, par exemple, est un mot qui appartient au langage courant, mais évidemment aussi un mot du vocabulaire technique de l'ornithologie. M. Coseriu, pourvu que je comprenne bien, éliminerait probablement ce mot-là, il dirait qu'il s'agit là d'une pure nomenclature, dépourvue de structuration. $\mathrm{La}$ première définition : "oiseau qui chante très bien ", me paraît évidemment un peu bête, mais je crois que c'est tout ce qu'on peut dire si l'on veut décrire le concept, la notion de rossignol, l'idée de rossignol telle 
qu'elle est vivante dans le langage courant. Prenez la définition du Petit Larousse, elle va, à mon avis, beaucoup trop loin : " oiseau passereau à plumage brun clair séjournant l'été dans nos bois et nos parcs et dont le mâle est un chanteur remarquable $»$ c'est déjà une information, c'est une connaissance qu'on peut avoir, mais je suis certain que la plupart des locuteurs ne l'ont pas. Quand on abandonne le domaine du langage courant, évidemment, il faut donner des descriptions, des définitions beaucoup plus précises et on entre alors pleinement dans la science. Du point de vue scientifique, du point de vue de l'ornithologie, la définition même du Petit Larousse reste très rudimentaire. Dans le langage courant, il faut décrire ce qu'on trouve dans les idées des locuteurs moyens. Dans le langage scientifique, il faut préciser et il n’y a pas de limites à la précision.

Réponse : Je crois que la limite entre le langage courant et le langage technique est extrêmement difficile à préciser. Il n'y a pas vraiment de limites; nous voyons tous les jours dans nos textes l'emploi de mots du langage courant qui couvrent une notion tout à fait nouvelle. C'est là justement où apparaît la difficulté de la normalisation du langage. Quand, par exemple, les instituts de normalisation prescrivent à un certain moment qu'on peut seulement employer tel et tel vocabulaire, ils ne peuvent pas tenir compte des particularités d'expression de M. X., qui innove tout d'un coup. M. X. doit rédiger son texte à la hâte parce que, forcément, les personnes qui rédigent des documents souvent après de longues et difficiles recherches, sont arrivées à la date limite où il leur faut absolument livrer le rapport. Il ne peut chercher très longtemps, il va prendre les mots qui lui viennent immédiatement à l'esprit. Vous avez aussi parlé de nomenclature. S'il y a une chose difficile à traduire, ce sont justement ces affreuses nomenclatures douanières et les nomenclatures pour les taxes, les impôts, etc., parce qu'il y a trop d'impon- dérables. Une chaise reste toujours une chaise, mais, pour des raisons de rentabilité ou autres, elle pourra prendre une appellation plus prestigieuse. Là c'est très difficile parce qu'il faut rendre sans point de repère un mot isolé d'une langue par un autre mot isolé d'une autre langue.

M. Guy Richard : Je voudrais préciser un petit point. Lorsqu'un institut de normalisation établit un vocabulaire technique, il se garde bien évidemment de déborder sur les termes du langage courant à moins que ce ne soit vraiment nécessaire. Lorsque nous rencontrons un terme du langage courant, nous renvoyons à un dictionnaire d'usage et nous ne nous en préoccupons pas. Je voudrais signaler aussi que, et c'est ce qui différencie peut-être messieurs les traducteurs, des techniciens et des ingénieurs, c'est que nous nous occupons strictement d'un langage délimité à nos domaines d'application, à nos domaines d'emploi. C'est là, je crois, que se situe la différence fondamentale entre vos préoccupations et les nôtres. Ce qui nous importe, lorsqu'un ingénieur anglais, un ingénieur britannique, un ingénieur français parlent d'une densité - ce qui est une notion tout à fait abstraite - c'est que les uns et les autres mettent le même contenu sous le terme qui nous sert à l'exprimer. Je ne rappellerai pas l'histoire banale des faux amis, mais enfin dans les débats internationaux pendant très longtemps nous nous sommes mal compris parce que la densité anglaise n'avait pas la même teneur, et je dirais pas seulement sémantique mais intellectuelle, que la densité française. La densité anglaise correspond exactement à ce que nous appelons nous, Français, une masse volumique. Par conséquent, c'est sur ce point précis que nous différons et ce que nous ambitionnons, c'est justement de donner au contenu de nos termes - et c'est l'objet de la définition - une valeur précise et qui, pour n'être pas ne varietur, dure quand même assez longtemps pour que l'application de cette notion à la technique ou à la science concernée soit valable. 